\title{
Cyclic Variation in the Solar Lower Atmosphere
}

\author{
C. Fang, Y. X. Zhang and M. D. Ding \\ Department of Astronomy, Nanjing University, Nanjing, China
}

W. C. Livingston

National Solar Observatory, Tucson, AZ85726-6732, USA

\begin{abstract}
Ca II $\mathrm{K}$ line has been measured regularly nearly every month since 1974 at Kitt Peak. Our study on the data of CaII K profiles over two solar cycles indicates that in center disc spectra, the distance of the $K_{1}$ component of the CaII $\mathrm{K}$ line from the line center and its intensity show periodical variations, but has a time delay of 1.2 years with respect to the sunspot number. Non-LTE computation implies a cyclic temperature variation of about $17 \mathrm{~K}$ of the temperature minimum region (TMR) in the quiet-Sun atmosphere and a cyclic variation of about $15-20 \mathrm{~km}$ of the position of the TMR.
\end{abstract}

\section{Introduction}

To fully understand our Sun, quiet-Sun regions are important. Some early attention has been paid to the variability of the photospheric network (Muller \& Roudier 1984), the magnetic flux (LaBonte \& Robert 1982) in the quiet-Sun regions, and the EUV variability (e.g. Rabin \& Dowdy 1992).

It is well known that the minimum intensity in the Ca II K line profile, $\mathrm{K}_{1}$, is formed mainly in the temperature minimum region (TMR). According to our study (Fang et al. 1986), the farther the distance of $K_{1}$ from the line center, the lower the position of the TMR would be; the stronger the intensity of $K_{1}$, the higher the temperature of the TMR. Thus, we used Ca II K line profiles at the solar disc center $\left(1^{\prime} \times 3^{\prime}\right)$ and measured $I_{\mathrm{K} 1}$, the average intensity of the red $K_{1}$ and the blue $K_{1}$ and $D_{\mathrm{K} 1}$, the distance between the red $K_{1}$ and the blue $K_{1}$.

\section{Observations}

Since 1974, a program has performed at Kitt Peak to measure the variability of the solar illuminance (full disk) spectrum over the 11 year solar cycle. Somewhat less frequently observations were made in $\mathrm{Ca}$ II $\mathrm{H}$ and $\mathrm{K}$ lines at disk center (White \& Livingston 1981). High resolution line profiles of Ca II K line were obtained by using the McMath double-pass spectrometer. In order to reduce low frequency noise, 30 rapid scans are averaged to obtain the final spectrum. Figure 1 shows the cyclic variation of $I_{\mathrm{K} 1}$ and $D_{\mathrm{K} 1}$ for the CaII $\mathrm{K}$ line at or near the solar disc center. 

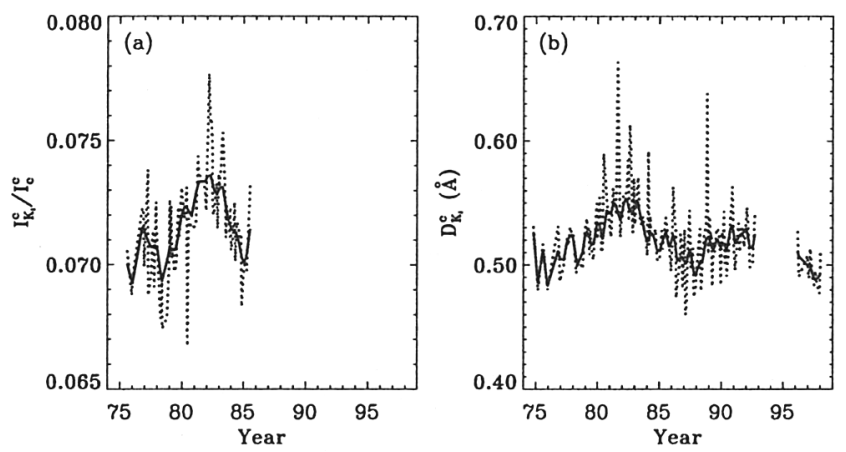

Figure 1. Cyclic variations of (a) the average relative intensity of the red $K_{1}$ and the blue $K_{1}$ of Ca II $K$ line profiles and (b) The distance between the red $K_{1}$ and the blue $K_{1}$ of the profiles. The solid lines are smoothed from the dotted lines of originally obsereved data. All profiles were observed at or near the solar disc center.

\section{Data Analyses}

\subsection{Periodicity Analysis}

A periodicity can be seen by visual inspection. Since the data are non-uniformly sampled, Lomb's algorithm (Lomb 1976; Scargle 1982) was used. Our calculation reveals that A period of 10.9 years for $D_{\mathrm{K} 1}$ and a period of 8.4 years for $I_{\mathrm{K} 1}$ (Figure 2). It should be mentioned that no calibrated observations are available for the last ten years and thus the 8.4 year period for $I_{\mathrm{K} 1}$ is only an approximate.

\subsection{Correlation Analysis}

To search for the relationships between these different data sets, cross-correlation analysis is performed. It is found that both for $D_{\mathrm{K} 1}$ and $I_{\mathrm{K} 1}$ the correlation coefficients peak at a time delay of around 15 months (1.2 yrs) with respect to $N_{\mathrm{SP}}$. No such phase shift was found in the case of solar full disc integrated $\mathrm{K}$ line profiles.

\subsection{Non-LTE Computations}

To know how temperature and column mass change in the quiet-Sun TMR during the solar cycle, non-LTE computations are needed. We iteratively solved the statistical equilibrium and radiative transfer equations as well as the hydrostatic equilibrium. The VAL3C model (Vernazza et al. 1981) was taken as a basis. Two dimensional grids of models were constructed based on VAL3C: In the first dimention $\Delta T$ is assumed to ocurr at the TMR, while $\Delta m=0$; In the second dimention $\Delta m$ is assumed, while $\Delta T=0$. For a given pair of $\Delta T$ and $\Delta m$, a new atmospheric model is obtained. Spectra from these models were then computed. 

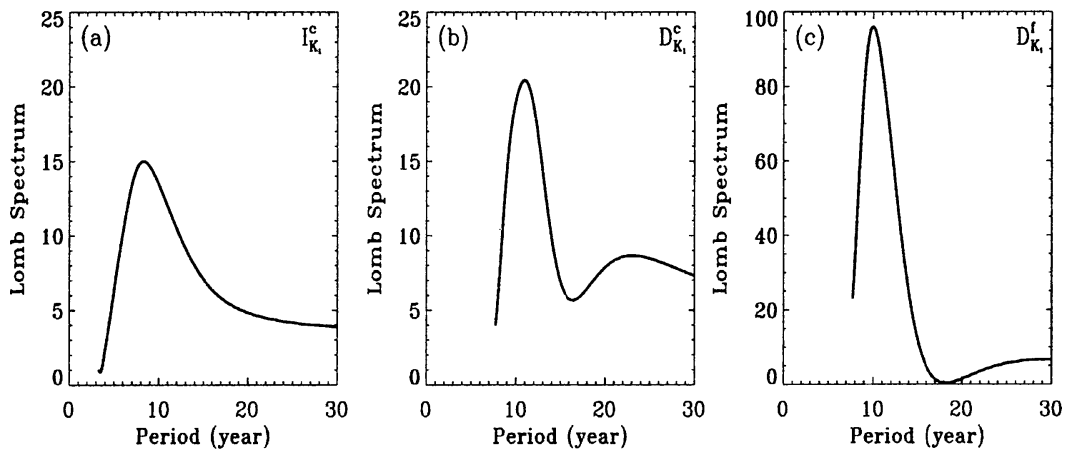

Figure 2. Lomb spectra showing possible periodicity of (a) $I_{\mathrm{K} 1}^{c}(8.4$ year), (b) $D_{\mathrm{K} 1}^{c}$ (10.9 year) and (c) $D_{\mathrm{K} 1}^{f}(10.0$ year).

By linear fitting, we obtained a relationships between the observed variations of $I_{\mathrm{K} 1}$ and $D_{\mathrm{K} 1}$ and the values of $\Delta T$ and $\Delta m$. From this formulae, it was found that during a solar cycle: $\Delta T \simeq 17 \mathrm{~K}$, and $\Delta m \simeq 1.7 \times 10^{-2} \mathrm{~g} \mathrm{~cm}^{-2}$.

\section{Conclusion}

(1) In the TMR the quiet-Sun atmosphere varies with a period that is comparable to that of the solar activity cycle. During a solar cycle, $\Delta T \simeq 17 \mathrm{~K}$, and $\Delta m \simeq 1.7 \times 10^{-2} \mathrm{~g} \mathrm{~cm}^{-2}$.

(2) The time delay between the quiet-Sun behavior and that of the active Sun is about 15 months. It suggests an unrevealed interactive mechanism between the quiet atmosphere and the plages or the active networks.

Acknowledgments. This work was supported by a fund from the National Natural Science Foundation (No. 49990451) and a fund from the Doctoral Program of the Ministry of Education of China. We are also grateful to NSO for providing the data.

\section{References}

Fang et al. 1986 In: The Lower Atmosphere of Solar Flares, ed. D. F. Neidig, NSO, Sunspot, New Mexico, 117

LaBonte, B. J., \& Robert, H. 1982 Solar Phys., 80, 15

Lomb, N. R. 1976 Ap\&SS, 39, 447

Muller, M., \& Roudier, T. 1984 Solar Phys., 94, 33

Rabin, D., \& Dowdy, J. F., Jr. 1992 ApJ, 398, 665

Scargle, J. D. 1982 ApJ, 263, 835

Vernazza, J. E., Avrett, E. H., \& Loeser, R. 1981 ApJS, 45, 635

White, O. R., \& Livingston, W. C. 1981 ApJ, 249, 798 